\title{
A two-well method to evaluate transverse dispersivity for tracer tests in a radially convergent flow field
}

\author{
Jui-Sheng Chen ${ }^{\mathrm{a}}$, Chia-Shyun Chen ${ }^{\mathrm{b}}$, Hwa-Sheng Gau ${ }^{\mathrm{c}}$, Chen-Wuing Liu ${ }^{\mathrm{d} \text {,* }}$ \\ ${ }^{a}$ Department of Environmental Engineering and Sanitation, Foo-Yin Institute of Technology, Kaohsiung, Taiwan, ROC \\ ${ }^{\mathrm{b}}$ Institute of Applied Geology, National Central University, Taoyuan, Taiwan, ROC \\ ${ }^{\mathrm{c}}$ Division of Geotechnique Engineering, Institute of Planning and Hydraulic Research, T.P.G.W.R.D., Taichung, Taiwan, ROC \\ ${ }^{\mathrm{d}}$ Department of Agricultural Engineering, National Taiwan University, Taipei, Taiwan, ROC
}

Received 23 December 1998; accepted 14 July 1999

\begin{abstract}
This work presents a two-well method to evaluate transverse dispersion coefficients for tracer tests conducted in a radially convergent flow field. A two-dimensional mathematical model is derived and applied to illustrate how transverse dispersion influences tracer transport in a convergent radial tracer test. A curve-fitting procedure is proposed to evaluate simultaneously the longitudinal and transverse dispersivities. A hypothetical experiment is used to demonstrate the application of the method. The proposed method allows one to evaluate the transverse dispersivity from field tracer tests, as long as the observed breakthrough curves at the pumping well and an observing well are known. (C) 1999 Elsevier Science B.V. All rights reserved.
\end{abstract}

Keywords: Tracer test; Transverse dispersion; Radially convergent flow field; Laplace transform finite difference method; Finite Fourier cosine transform

\section{Introduction}

Tracer tests are performed by introducing an easily measured chemical tracer into the aquifer and observing it in another well. Tracer tests attempt to determine and characterize the solute transport parameters, such as kinematic aquifer porosity, dispersion tensor and the hydrogeological properties of an aquifer. In numerical modeling, the dispersion coefficient is the major input solute transport parameter that needs to be estimated in the field condition. Under many circumstances, tracer experiments in a radially convergent flow field, are preferred over other methods. Many investigators have extensively

* Corresponding author. Tel.: + 886-2-362-5462; fax: + 88622363-9557. developed the one-dimensional mathematical models in cylindrical coordinates to determine longitudinal dispersivity from the convergent tracer test. (Sauty, 1980; Carrera and Walters, 1985; Guvanasen and Guvanasen, 1987; Moench, 1989; Wang and Crampon, 1995; Chen et al., 1996). They assumed that effects due to transverse dispersion do not appear at the pumping well. These authors worked on the breakthrough curve at pumping well, without benefitting from information on other observation wells.

Consider the one-dimensional model in cylindrical coordinates developed in previous investigations. The one-dimensional model approach implies that once all streamlines merge in the pumping well, the effect of transverse dispersion is not visible. However, because of variations in microscopic velocity (in both magnitude and direction, within each pore channel and from 


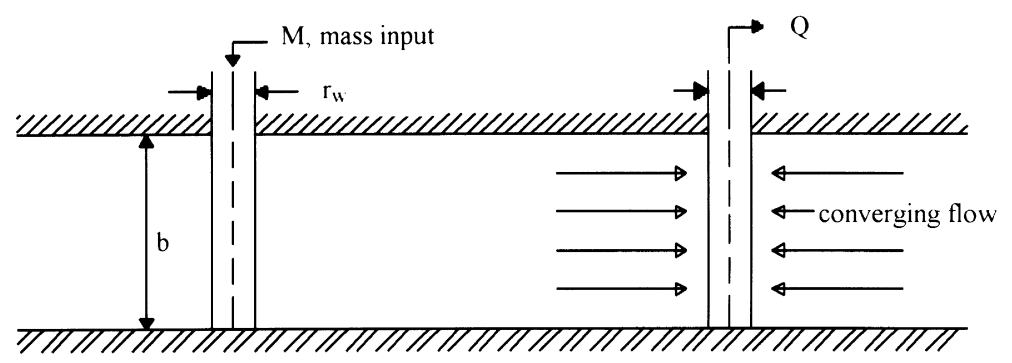

(A)

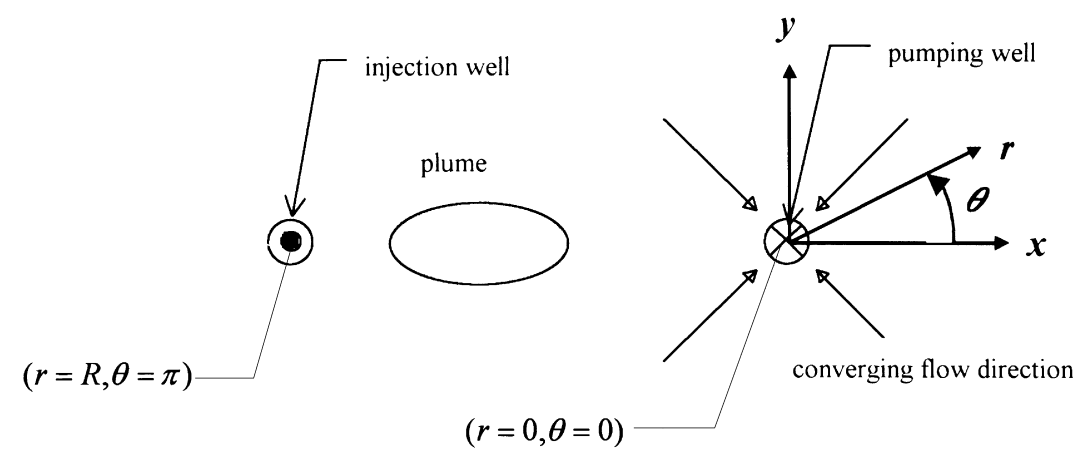

(B)

Fig. 1. Schematic diagram of radially convergent tracer test: (A) side view on top; (B) plane view on bottom.

one channel to another) force water molecules to move along tortuous flow paths which may be parallel or normal to the average flow direction, and cause spreading of the solute. Leij and Dane (1991) indicated that applying the one-dimensional advectiondispersion equation in Cartesian coordinates to determine longitudinal dispersion coefficients in a heterogeneous medium, with concentration gradient in the transverse direction, leads to erroneous values for longitudinal dispersion, since the profile cannot be described by Gaussian spreading. A similar conclusion was reached by Domenico and Robbins (1984).

Sauty (1980) pointed out that the one-dimensional model does not yield transverse dispersivity. However, the magnitude of the transverse dispersion coefficient influences both the region to which the pollution is extended, and the intensity of the pollution. Increasing the transverse dispersion coefficient causes the plume to spread out over a large area. If low levels of contaminant are acceptable, increasing the transverse dispersion coefficient is highly desirable. Substances which threaten groundwater quality, even at a low concentration, and must subsequently be removed, should be confined to the smallest possible region and therefore have a low transverse dispersion coefficient. The above reasons account for the importance of determination of the transverse dispersion coefficient.

In a related work, Guvanasen and Guvanasen (1987) considered transverse dispersion in their model, to describe how the geometrical configuration of tracer plumes influences the tracer test by deriving an approximate two-dimensional, semi-analytical solution in streamline and equipotential coordinates. Their solution is appropriate for breakthrough curve generation only because the advection term, longitudinal and transverse dispersion terms of the governing equation are linearized from the injection point to the 
Table 1

The variables used in this study and their meanings and dimensions

\begin{tabular}{lll}
\hline Variable & Meaning & Dimension \\
\hline$r_{c}$ & The radius of pumping well & {$[\mathrm{L}]$} \\
$Q$ & Pumping rate & {$\left[\mathrm{L}^{3} / \mathrm{T}\right]$} \\
$b$ & Aquifer thickness & {$[\mathrm{L}]$} \\
$n_{\mathrm{e}}$ & Effective porosity & {[]} \\
$A$ & $A=Q / 2 \pi b n_{\mathrm{e}}$ & {$\left[\mathrm{L}^{2} / \mathrm{T}\right]$} \\
$V$ & Effective porosity & {$[\mathrm{L} / \mathrm{T}]$} \\
$r_{\mathrm{w}}$ & The radius of injection borehole & {$[\mathrm{L}]$} \\
$R$ & The distance between pumping well and & {$[\mathrm{L}]$} \\
& injection well & \\
$C$ & Concentration & {$\left[\mathrm{M} / \mathrm{L}^{3}\right]$} \\
$a_{\mathrm{L}}$ & Longitudinal dispersivity & {$[\mathrm{L}]$} \\
$a_{\mathrm{T}}$ & Transverse dispersivity & {$[\mathrm{L}]$} \\
$r$ & Radial distance & {$[\mathrm{L}]$} \\
$\theta$ & Transverse angle & {$[\mathrm{L}]$} \\
$M$ & The injected tracer mass & {$[\mathrm{M}]$} \\
$\Delta r$ & $\Delta r=2 r_{\mathrm{w}}$ & {$[\mathrm{L}]$} \\
$\Delta \theta$ & $\Delta \theta=\pi r_{\mathrm{w}} / 2 R$ & {[]} \\
$H(\cdot)$ & Heaviside unit step function & {[]} \\
$\delta(\cdot)$ & Dirac delta function & {$\left[\mathrm{T}{ }^{-1}\right]$} \\
$\tau$ & Dimensionless time & {[]} \\
$\rho$ & Dimensionless radial distance & {[]} \\
$P e$ & Peclet number, $P e=R / a_{\mathrm{L}}$ & {[]} \\
$C_{\mathrm{r}}$ & $C_{r}=M / R b n_{\mathrm{e}} \Delta r \Delta \theta$ & {$\left[\mathrm{M} / \mathrm{L}^{3}\right]$} \\
$X$ & Dimensionless ratio of dispersivity & {[]} \\
$L$ or $L^{-1}$ & Laplace transform or its inverse & \\
$F$ or $F^{-1}$ & Finite Fourier cosine transform or its inverse & \\
\hline & &
\end{tabular}

observation point in order to eliminate the dependency of velocity, longitudinal dispersion and transverse dispersion on radial distance. The three defined parameters must be adjusted to reflect accurately the spatial position of all observation points, in order to obtain the spatial distribution of concentration at a specific time. Adjustment of the parameters is a rather complex process because a numerical experiment must be performed to determine the range within which the parameters should be applied. Guvanasen and Guvanasen (1987) observed a discrepancy between their approximate analytical solution and a verified finite element solution for a small Peclet number.

Relatively few field values of the transverse coefficient obtained from natural-gradient dispersion tests, have been reported (Sudicky and Cherry, 1979; Sudicky et al., 1983). For instance, Moltyaner and Killey (1988) obtained a vertical-transverse dispersivity of $0.0001-0.0019 \mathrm{~m}$ for natural-gradient tests at the Chalk River Nuclear Laboratories in Ontario.
Therefore, field experiments are necessary first and numerical simulators could interpret them as a first step.

In light of above developments, this study derived a numerical solution for a two-dimensional model, so that the effects of longitudinal and transverse dispersion could be simultaneously estimated. A two-well method, which applied a curve-fitting procedure involving a type curve at the pumping well and a breakthrough curve at one of the observation points, is proposed to determine the transverse dispersivity for a tracer test. The developed two-well method is useful in determining the longitudinal and transverse dispersivities for the field tracer test in a radially convergent flow field.

\section{Mathematical model}

The dispersion problem of a tracer test in a radially convergent flow field is considered. Fig. 1 depicts the conceptual configuration. The flow field is generated by a fully penetrating well of radius $r_{\mathrm{c}}$ located along the vertical axis at $r=0$, and pumping fluid at a constant volume rate $Q$ from a homogeneous, isotropic aquifer of infinite horizontal extent. (Table 1 lists all variables, their meanings and dimensions). The average pore velocity, $V$, in the radial direction is described by

$V=-\frac{A}{r}$

where $A=Q / 2 \pi b n_{\mathrm{e}}$. Also, $b$ and $n_{\mathrm{e}}$ represent the aquifer thickness and effective porosity, respectively.

When a field test is started, once water levels are stabilized, a tracer is introduced into the injection borehole of radius $r_{\mathrm{w}}$, located at $r=R, \theta=\pi$ and flows out of the injection well borehole. For mathematical convenience, the initial tracer is assumed to be uniformly distributed on a cylinder, centered at the injection well. The packed interval's thickness equals the aquifer's thickness. Fig. 1 also indicates that the area of the tracer plume $\pi r_{\mathrm{w}}^{2}$ is approximately equal to the area element $r \Delta r \Delta \theta$ in cylindrical coordinates. The injected tracers are transported within the aquifer by advection, mechanical dispersion and molecular diffusion. Mechanical dispersion consists of longitudinal, vertical-transverse and horizontal-transverse 
dispersion. Solutes move along the advective flow path by advection and longitudinal dispersion. Horizontal-transverse and vertical-transverse dispersion cause solute to spread and deviate from the advective flow path. For simplicity, concentrations in the aquifer are taken as an average value over aquifer thickness. Correspondingly, only horizontal-transverse dispersion is considered for the transverse dispersion.

We assume that longitudinal and transverse mechanical dispersion occurs in accordance with Fick's law, and that both the longitudinal and transverse dispersion coefficient are proportional to the velocity. The effects of molecular diffusion are assumed to be markedly smaller than those of mechanical dispersion. The configuration in Fig. 1 is symmetrical around the line passing through the pumping and injection wells. Therefore, only one half-plane is considered. The governing equation for the radially convergent dispersion with instantaneous slug injection at the injection well, can be formulated in cylindrical coordinates as

$$
\frac{a_{\mathrm{L}} A}{r} \frac{\partial^{2} C}{\partial r^{2}}+\frac{A}{r} \frac{\partial C}{\partial r}+\frac{a_{\mathrm{T}} A}{r^{3}} \frac{\partial^{2} C}{\partial \theta^{2}}+f(r) g(\theta) \delta(t)=\frac{\partial C}{\partial t}
$$

where

$f(r)=\frac{M}{R b n_{\mathrm{e}} \Delta r \Delta \theta}\left[H\left(R+\frac{\Delta r}{2}\right)-H\left(R-\frac{\Delta r}{2}\right)\right]$,

$g(\theta)=\left[H(\pi)-H\left(\pi-\frac{\Delta \theta}{2}\right)\right]$

where $M$ denotes the tracer mass, $\Delta r=2 r_{\mathrm{w}}, \Delta \theta=$ $\pi r_{\mathrm{w}} / 2 R, H(\cdot)$ represents the Heaviside unit step function, $\delta()$ is the Dirac delta function, and $a_{\mathrm{L}}$ and $a_{\mathrm{T}}$ denote the longitudinal and transverse dispersivity, respectively.

The aquifer's initial tracer concentration is assumed to be zero before starting the test:

$C(r, \theta, 0)=0 \quad r_{\mathrm{c}} \leq r<\infty$

The pumping well is treated as a mathematical sink. The concentration inside the wellbore is assumed to be instantaneously in equilibrium with the surrounding media. This assumption implies that during the pumping period, a zero concentration gradient exists at the interface between the well and its immediate adjacent aquifer at $r_{\mathrm{c}}$. Therefore, the boundary condition at $r_{\mathrm{c}}$ is prescribed as

$$
\frac{\partial C(r, \theta, t)}{\partial r}=0 \text { at } r=r_{\mathrm{c}} \quad t>0
$$

Regarding the numerical solution's accuracy, we select a computational domain $\left(r_{\mathrm{L}}, r_{\mathrm{L}}>R\right)$ which is superior to the well-piezometer distance $(R)$ as indicated by Wang and Crampon (1995). The other boundary condition in the $r$-direction, as required for a unique solution to Eq. (3), is imposed at $\left(r_{\mathrm{L}}\right)$ by stating

$$
\begin{gathered}
C(r, \theta, t)+a_{\mathrm{L}} \frac{\partial C(r, \theta, t)}{\partial r}=0 \\
\text { at } r=r_{\mathrm{L}}, \quad r_{\mathrm{L}}>R, \quad t>0
\end{gathered}
$$

where $r_{\mathrm{L}}$ is the limit distance.

The physics of the problem stipulates that $C$ is single-valued in the aquifer. In addition, $C$, besides being single-valued, is obviously continuous and symmetrical across $\theta=0, \theta=\pi$. Thus, the boundary conditions in the transverse direction are given by

$\frac{\partial C(r, 0, t)}{\partial \theta}=0$

$\frac{\partial C(r, \pi, t)}{\partial \theta}=0$

Let $\tau$ be the dimensionless time, $\rho$ be the dimensionless radial distance, and $X$ be the dimensionless ratio of dispersivity. The governing Eq. (3) can be reexpressed in the following form:

$\frac{1}{\rho} \frac{\partial^{2} C}{\partial \rho^{2}}+\frac{1}{\rho} \frac{\partial C}{\partial \rho}+\frac{X}{\rho^{3}} \frac{\partial^{2} C}{\partial \rho^{2}}+f_{1}(\rho) g(\theta) \delta(\tau)=\frac{\partial C}{\partial \tau}$

where $f_{1}(\rho)=C_{\mathrm{r}}[H(P e+\Delta \rho / 2)-H(P e-\Delta \rho / 2)], \rho$ $=r / a_{\mathrm{L}}, P e=R / a_{\mathrm{L}}, \rho_{\mathrm{c}}=r_{\mathrm{C}} / a_{\mathrm{L}}, \rho_{\mathrm{w}}=r_{\mathrm{w}} / a_{\mathrm{L}}, \tau=A t / a_{\mathrm{L}}$, $X=a_{T} / a_{\mathrm{L}}, C_{\mathrm{r}}=M / R b n_{\mathrm{e}} \Delta r \Delta \theta, P e$ is Peclet number, $C_{\mathrm{r}}$ is defined as the reference concentration.

Consequently, the initial and boundary conditions become

$C(\rho, \theta, 0)=0$

$\frac{\partial C\left(\rho_{\mathrm{c}}, \theta, \tau\right)}{\partial \rho}=0$ 


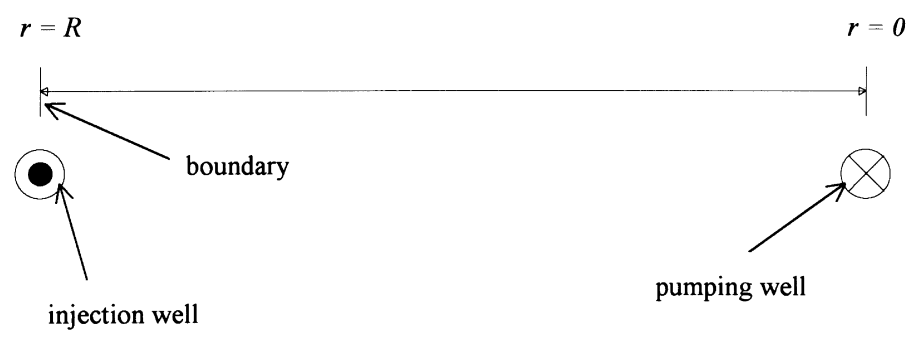

Fig. 2. Schematic diagram of computation domain for the verification case of a Laplace transform solution derived by Moench (1989), $r=0$ is pumping well, $r=R$ is the injection well and the outer boundary is set at the injection well.

$C\left(\rho_{\mathrm{L}}, \theta, \pi\right)+\frac{\partial C\left(\rho_{\mathrm{L}}, \theta, \tau\right)}{\partial \rho}=0$

$\frac{\partial C(\rho, 0, \tau)}{\partial \theta}=0$

$\frac{\partial C(\rho, \pi, \tau)}{\partial \theta}=0$

where $\rho_{\mathrm{L}}=r_{\mathrm{L}} / a_{\mathrm{L}}$.
In this study, we adopt the revised Laplace Transform Finite Difference (LTFD) method to solve the tracer test problem. The detailed derivation of the revised LTFD method is given in the Appendix A. The LTFD method was first developed by Moridis and Reddell (1991) to solve the partial differential equation of transient flow through porous media. The Laplace transform numerical method is preferred over conventional time-marching schemes. In this

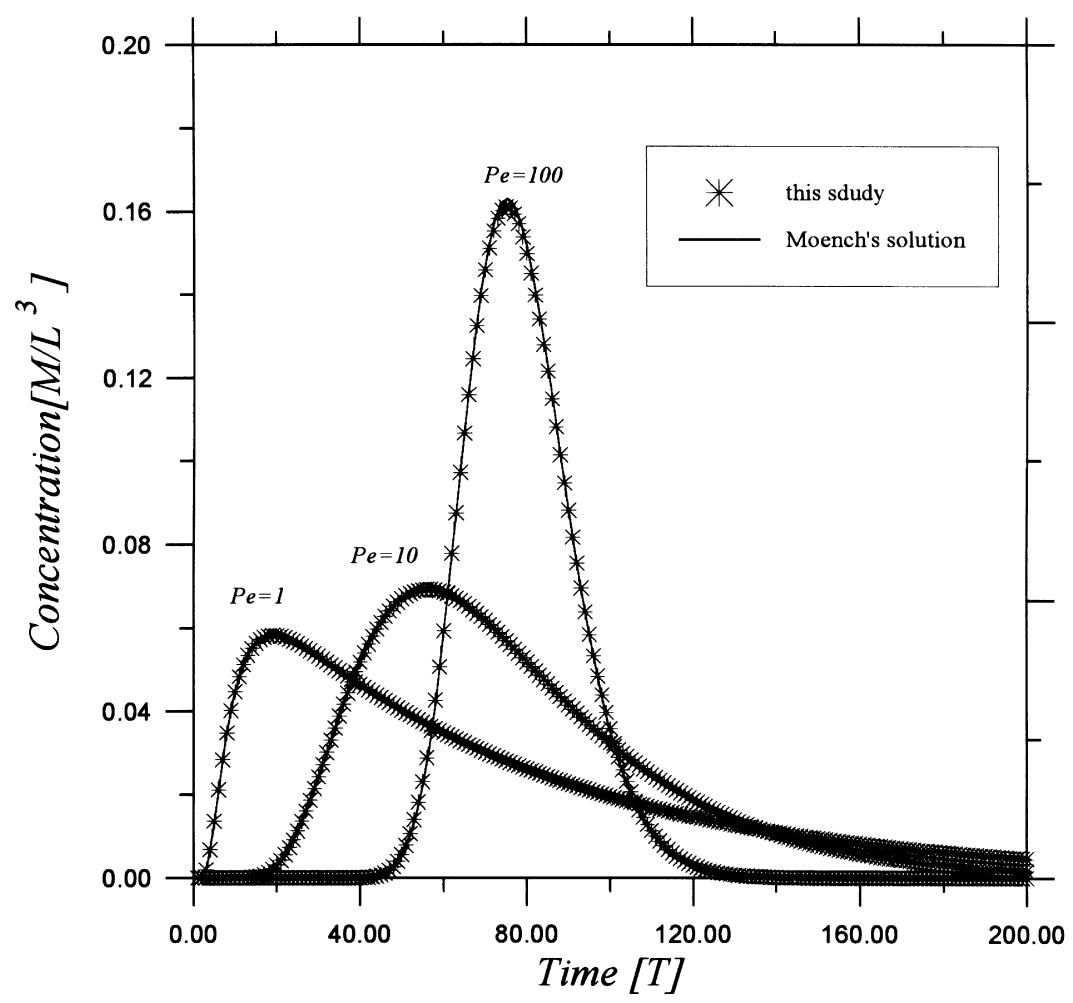

Fig. 3. Verification of dimensional breakthrough curves for the proposed model versus the Moench's (1989) model. 


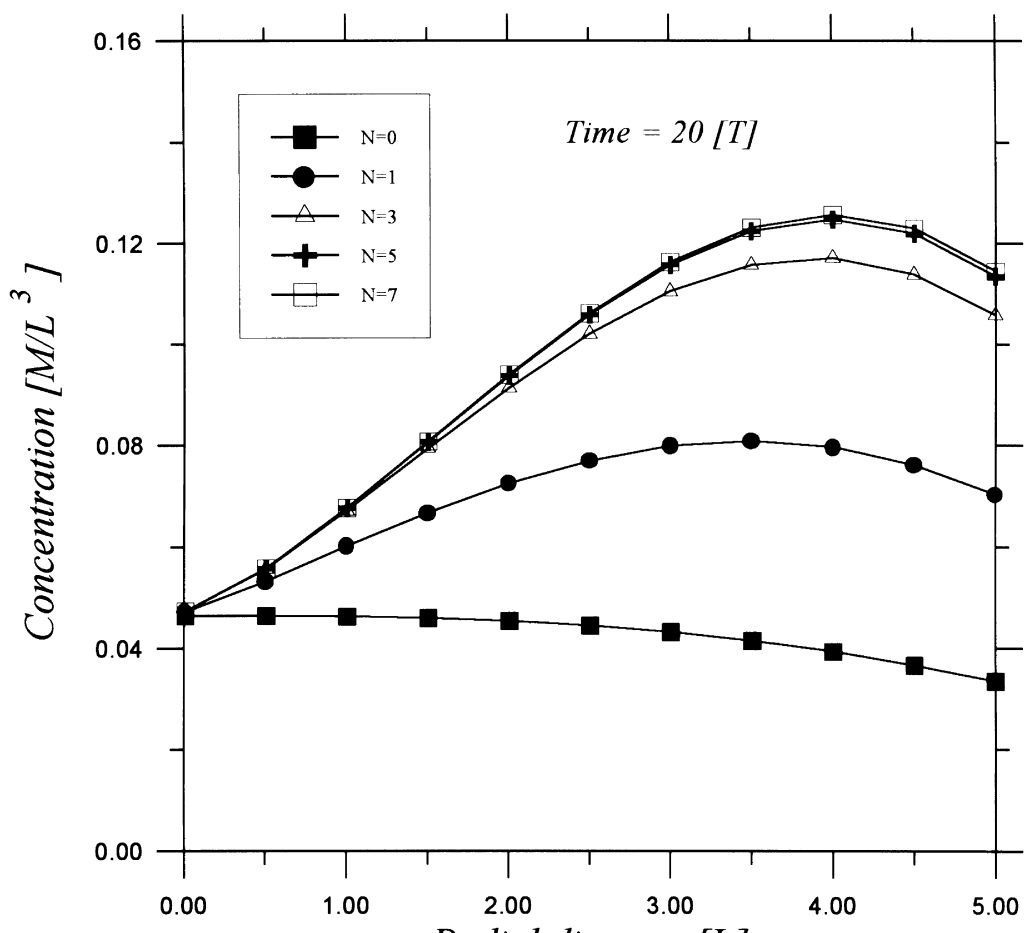

(a)

Radial distance [L]

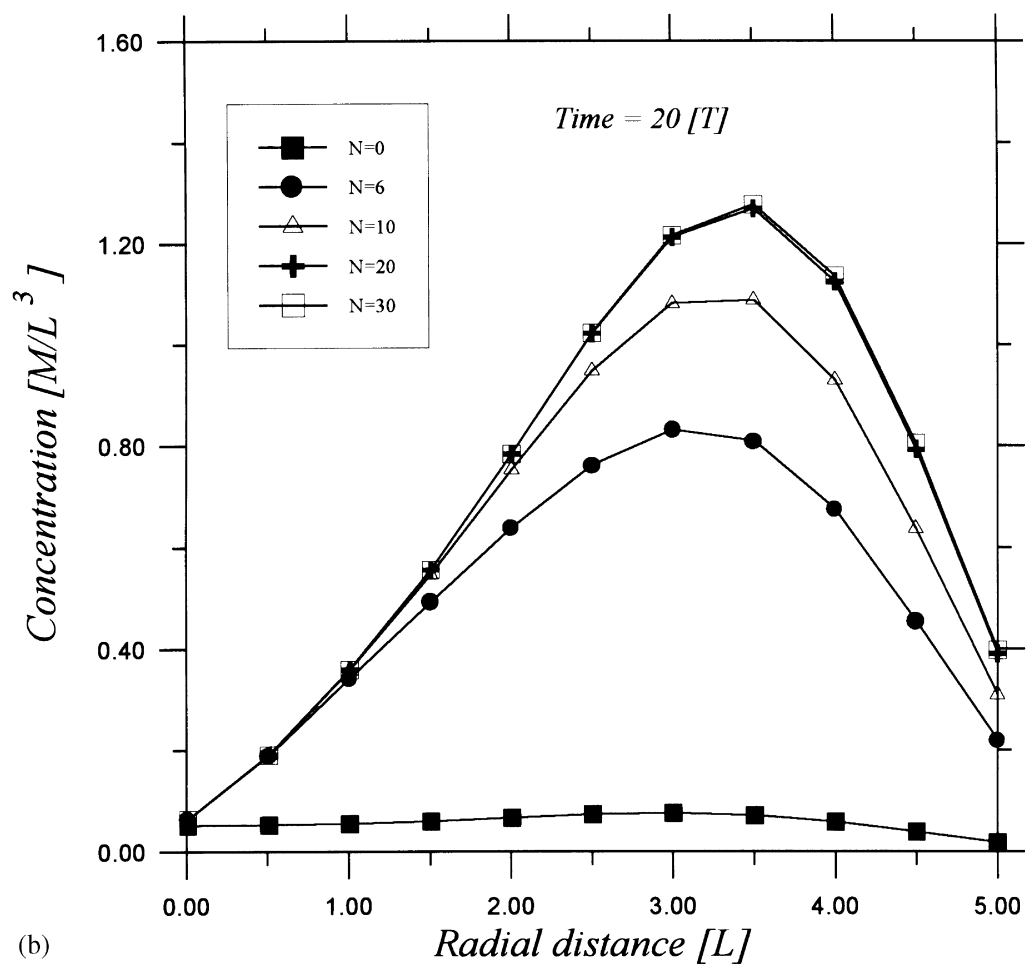




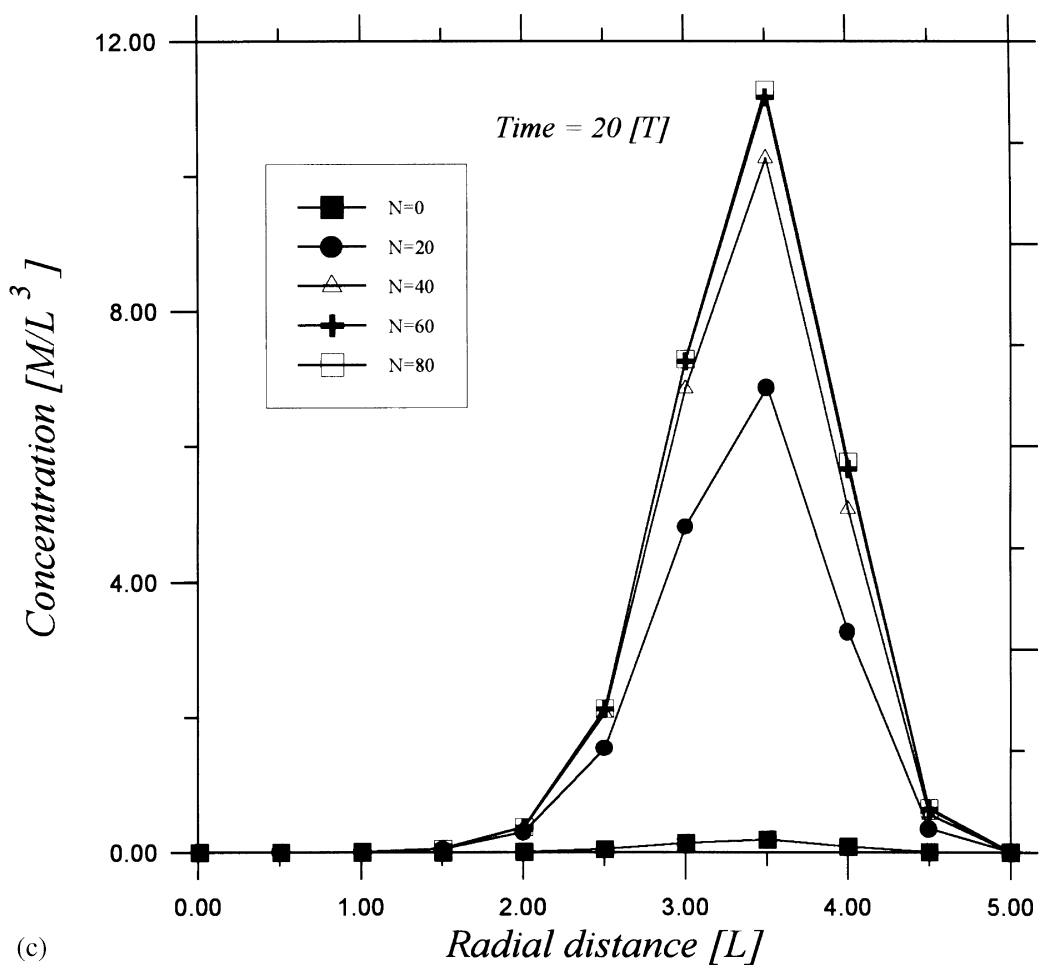

Fig. 4. (Continued).

approach, the Laplace transform is initially employed to eliminate the temporal derivative and obtain a frequency domain ( $p$-space) spatially distributed equation. The numerical method is then applied to obtain the $p$-space concentration at any spatial node. The solutions for different times are independent of each other, without evaluation of the solution at an intervening time. Also, the solution is exact in time, and the numerical error is restricted only to that introduced by discretizing space and evaluating the eigensystem (Sudicky, 1989; Moridis and Reddell, 1991; Sudicky and McLaren, 1992). In this study, we develop a revised LTFD solution which differs from the conventional LTFD technique. The space discretization is only performed in the radial direction. Before discretizing the radial distance, the finite Fourier cosine transform is performed with respect to the transverse angle variable. The proposed method is highly attractive since, in the resulting system of simultaneous equations, possible errors are diminished by an analytical transform which is used instead of the spatial discretization in a transverse direction. The required space-discretized nodes are only the number of discretizations in the radial direction. The method proposed here does not require any discretization in the transverse direction. After solving the Laplace-finite Fourier cosine domain concentration at node points using the conventional finite difference theory, the solution in the original domain is ultimately recovered using a Laplacian inversion and a finite Fourier cosine transform inversion.

Fig. 4. (a) The dependence of the numbers of the required series term $(N)$ for solution's convergence $(P e=1)$; (b) The dependence of the numbers of the required series term $(N)$ for solution's convergence $(P e=10)$; (c) The dependence of the numbers of the required series term $(N)$ for solution's convergence $(P e=100)$. 


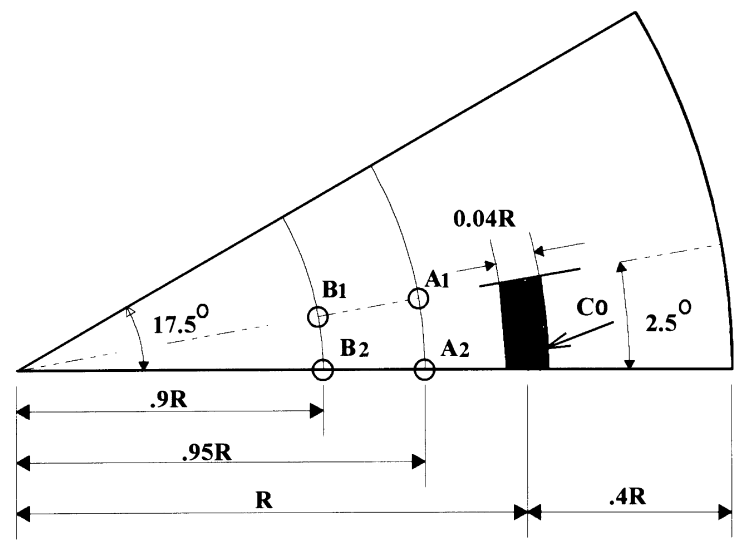

Fig. 5. Schematic diagram of domain discretized by finite elements (not to scale).

\section{Model verification}

Two verification problems are used to test the accuracy of the revised LTFD model for simulating the tracer transport behavior. A one-dimensional Laplace-domain analytical solution, the Moench (1989) solution, is the first problem of a convergent tracer test without upstream dispersion that we addressed. The revised LTFD solution is compared with the analytical solution, for verification purposes. The second problem is a two-dimensional, finite element solution used by Guvanasen and Guvanasen (1987) to verify their approximate solution.

\subsection{Case 1}

Case 1 is a test problem that Moench (1989) investigated, representing the one-dimensional, convergent

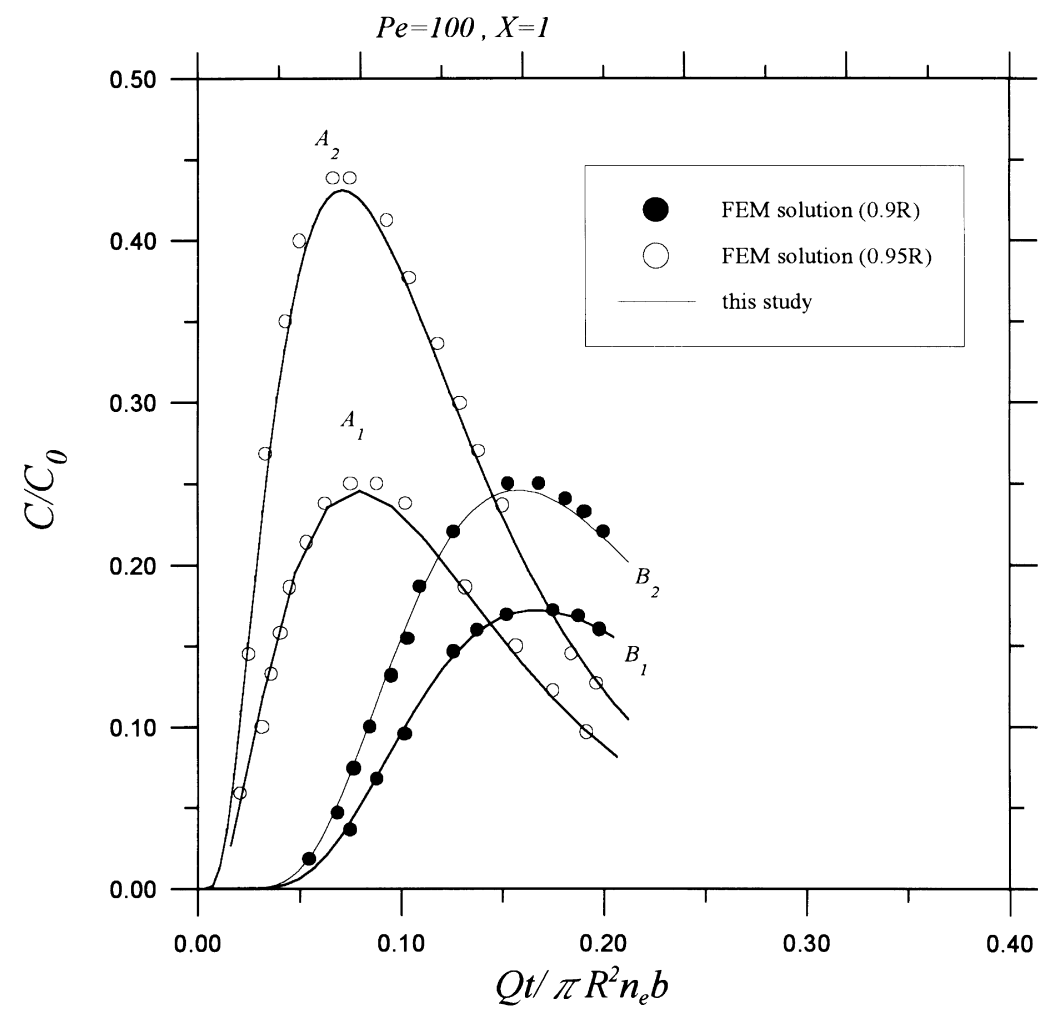

Fig. 6. Comparison between finite element solution (FEM) presented by Guvanasen and Guvanasen (1987) and the proposed model's solution of Dimensionless breakthrough curves at four observation points (see Fig. 5) between an initially rectangular tracer pulse and the pumping well.

Fig. 7. (a) Geometrical configuration of the developed steady state flow field of a convergent tracer test. (b) Concentration contours at a time $t=20[\mathrm{~T}]$. (c) Concentration contours at a time $t=40[\mathrm{~T}]$. (d) Concentration contours at a time $t=60[\mathrm{~T}]$. (e) Concentration contours at a time $t=80[\mathrm{~T}]$. (f) Concentration contours at a time $t=100[\mathrm{~T}]$. 

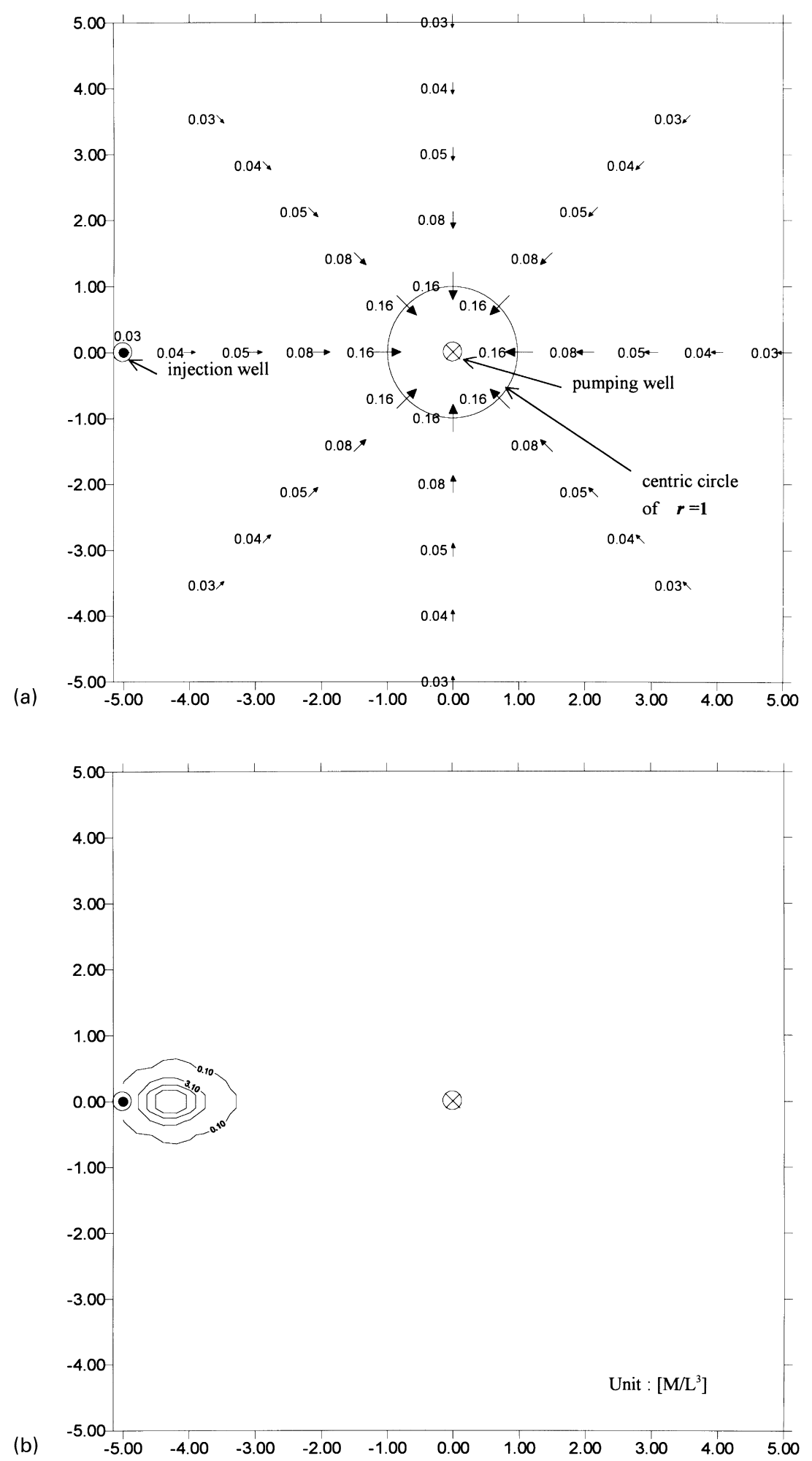

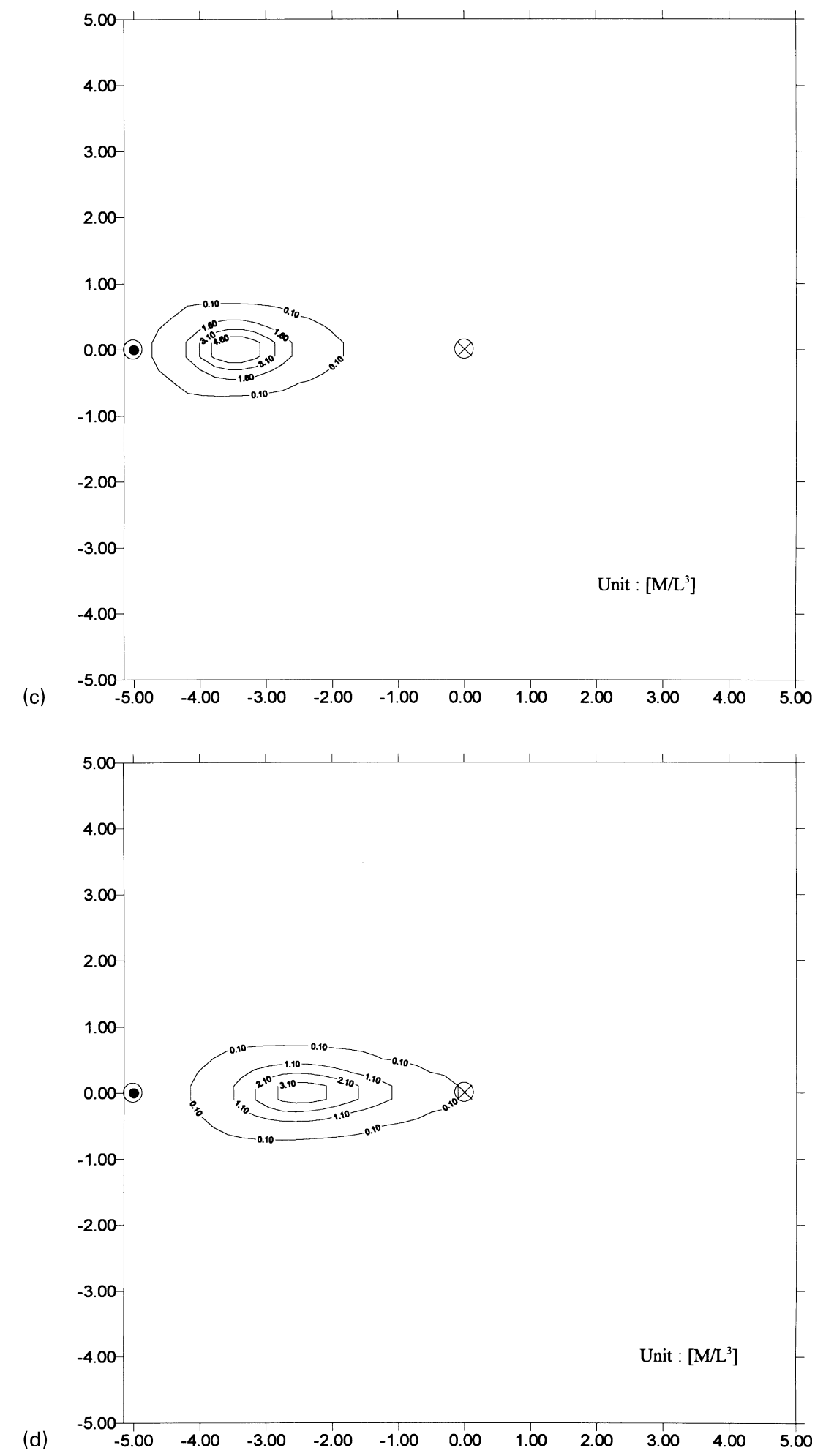

Fig. 7. (Continued). 

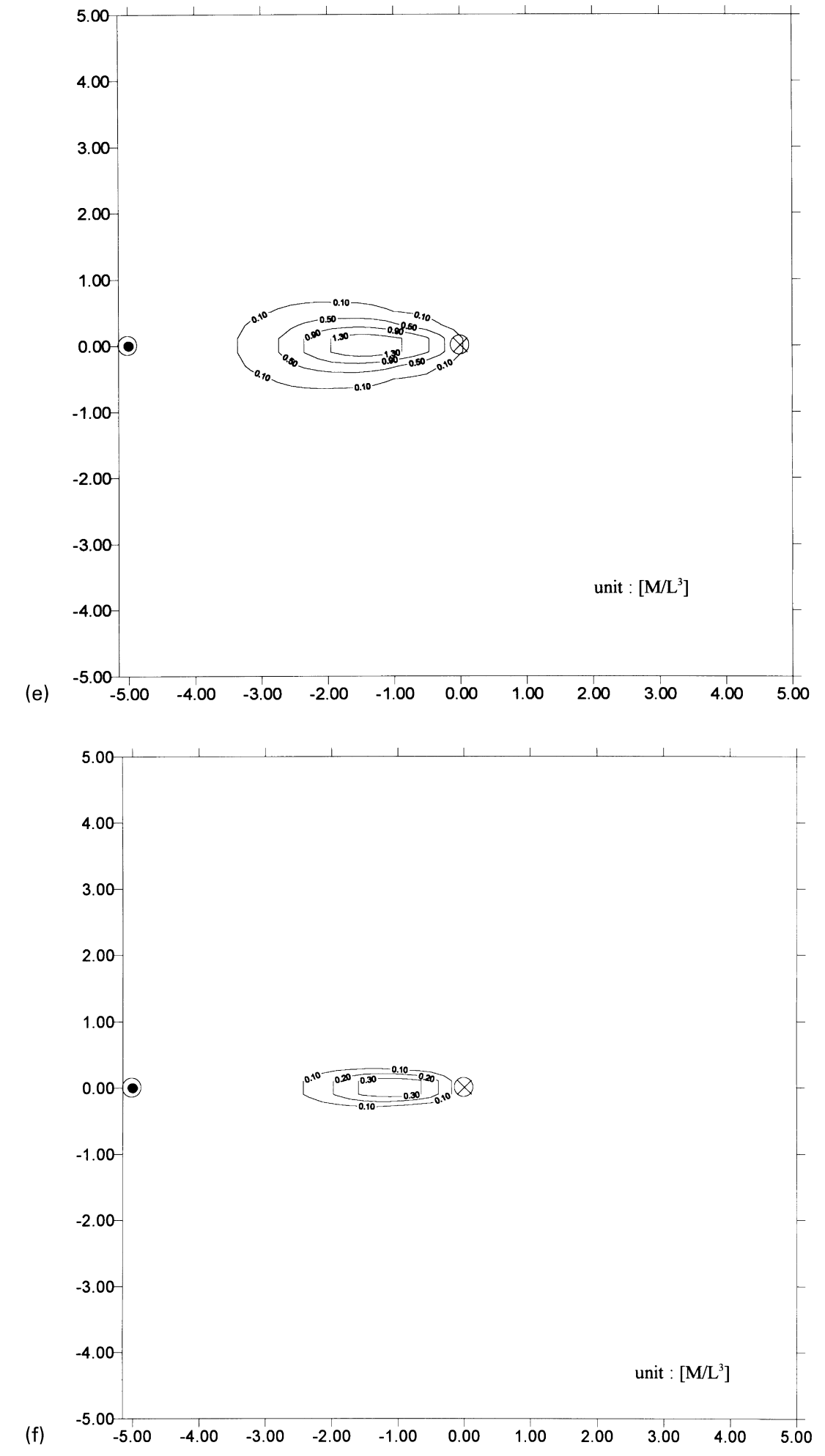

Fig. 7. (Continued). 

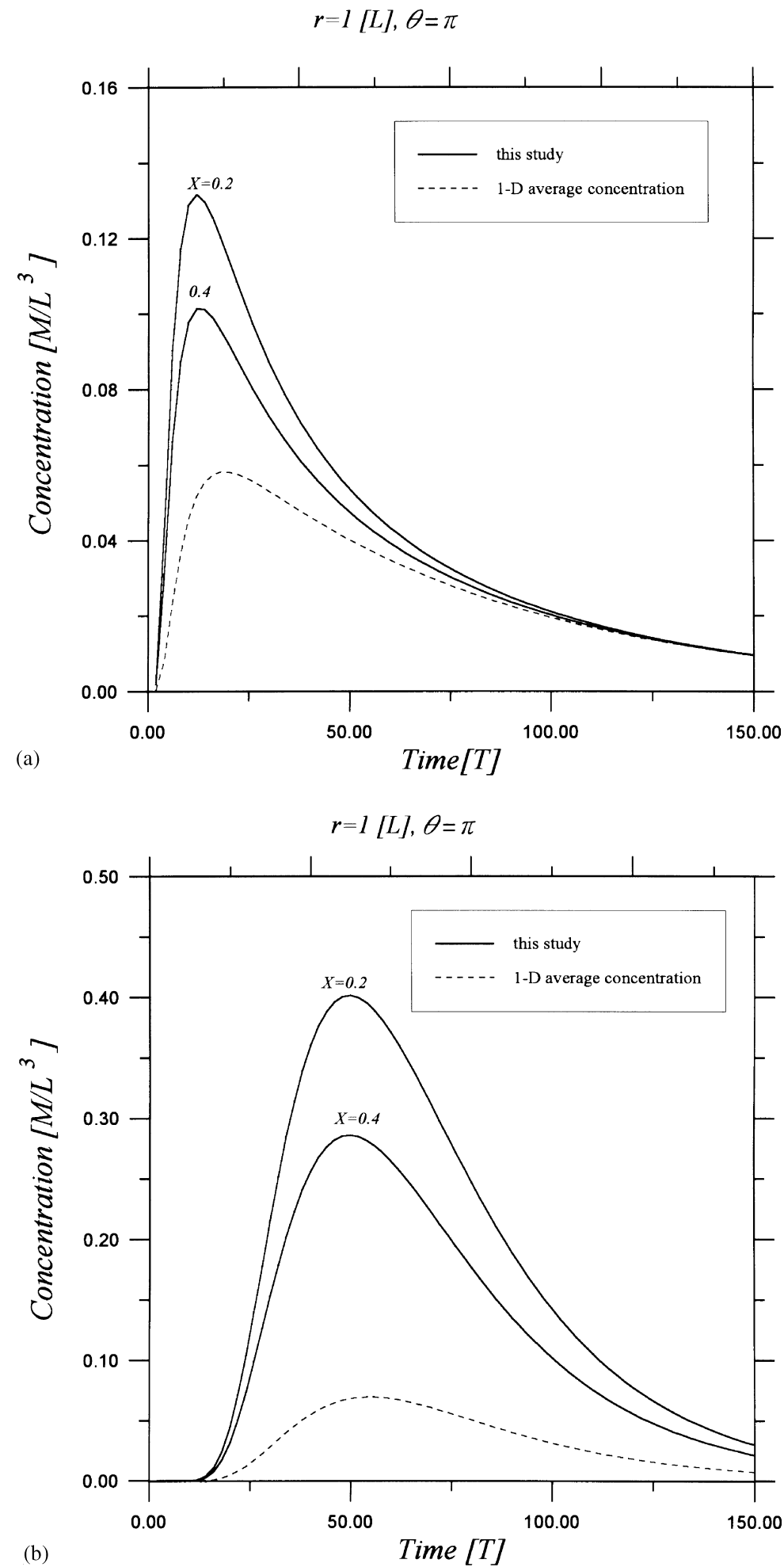


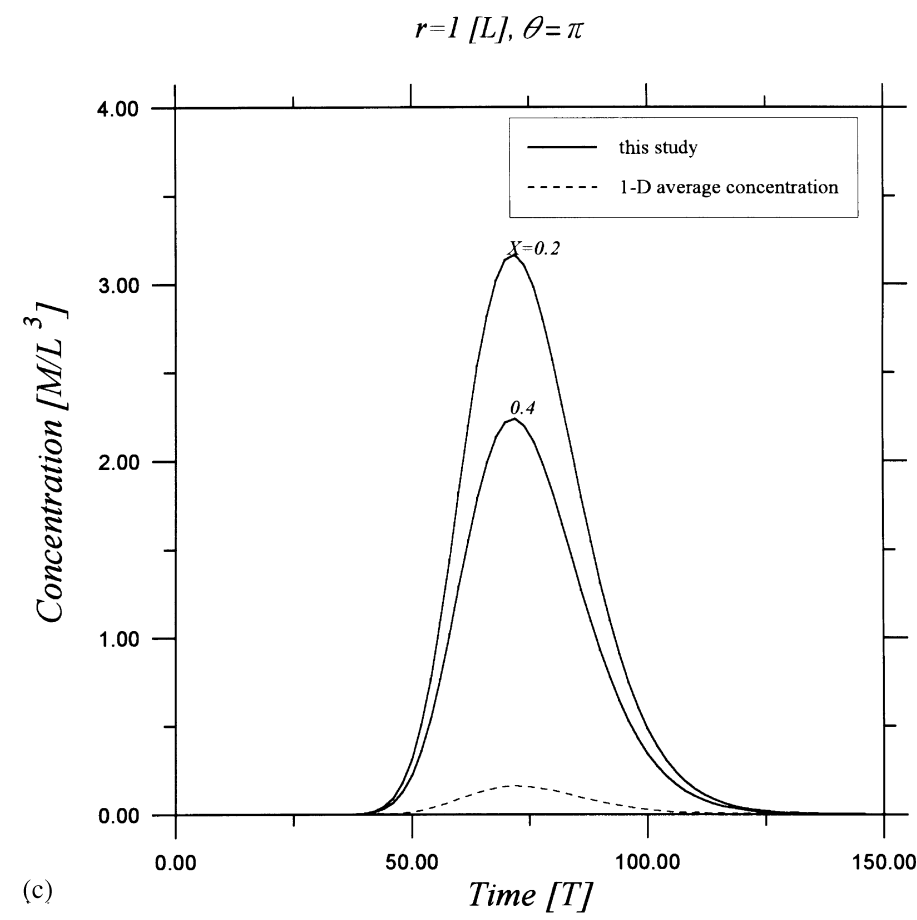

Fig. 8. (Continued).

radial dispersion transport. An input boundary is imposed at the injection well. The input boundary approach implies that no dispersive flux is allowed upstream of the injection well. Fig. 2 schematically depicts the test problem. In the current model, $r_{\mathrm{L}}$ is set to $R$. Although the model developed by Moench (1989) is mathematically one-dimensional, from a two-dimensional perspective, the solution is equivalent to that of an axisymmetric problem with an initial annulus plume. In the revised LTFD model, $\Delta \theta$ is set to $2 \pi$. The following hypothetical parameters are used: distance between centers of wells, 5 [L]; pumping rate $2\left[\mathrm{~L}^{3} \mathrm{~T}^{-1}\right]$; aquifer thickness, $10[\mathrm{~L}]$; effective porosity, 0.2 ; injected mass, $10[\mathrm{M}]$; radius of pumping well, $0.02[\mathrm{~L}]$; longitudinal dispersivity, $a_{\mathrm{L}}=5,0.5$ and $0.05[\mathrm{~L}]$ (that is, a Peclet number of
1,10 and 100); and transverse dispersivity, $a_{\mathrm{T}}=1$, 0.1 and $0.01[\mathrm{~L}]$ (that is, a dimensionless ratio of transverse over longitudinal dispersivity, where $X=$ $a_{\mathrm{T}} / a_{\mathrm{L}}$ is 0.2$)$. The fine-grid $(\Delta \rho=0.001)$ spacing in the radial flow direction is used to avoid numerical dispersion. The revised LTFD solution does not require discretization in the transverse direction.

Fig. 3 displays breakthrough curves at the pumped well for various Peclet numbers, and compares them to Moench's solution. According to this figure, concentrations obtained from the revised LTFD formulation agree with those obtained from the Laplace analytical solution.

To provide further insight into the solution's convergence, we investigate the dependence of the numbers of the required series term for the finite

Fig. 8. (a) Comparisons of dimensional breakthrough curves of dimensionless dispersivity ratio of $X=0.2$ and 0.4 at observation point at $r=1$ [L], $\theta=\pi$ for the two-dimensional solution of the proposed model and one-dimensional averaging solution in a hypothetical condition $(P e=$ 1). (b) Comparisons of dimensional breakthrough curves of dimensionless dispersivity ratio of $X=0.2$ and 0.4 at observation point at $r=1$ [L], $\theta=\pi$ for the two-dimensional solution of the proposed model and one-dimensional averaging solution in a hypothetical condition $(P e=10)$. (c) Comparisons of dimensional breakthrough curves of dimensionless dispersivity ratio of $X=0.2$ and 0.4 at observation point at $r=1$ [L], $\theta=\pi$ for the two-dimensional solution of the proposed model and one-dimensional averaging solution in a hypothetical condition $(P e=100)$. 

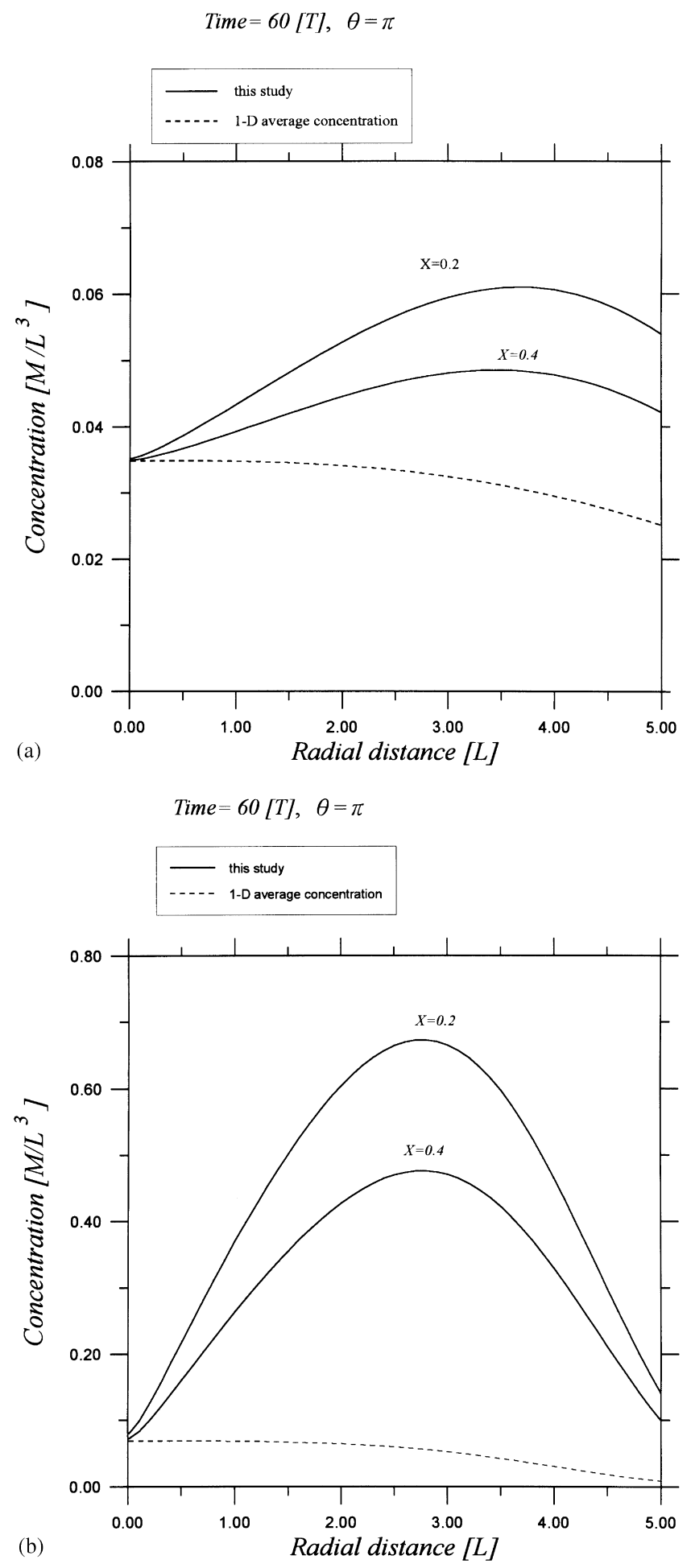


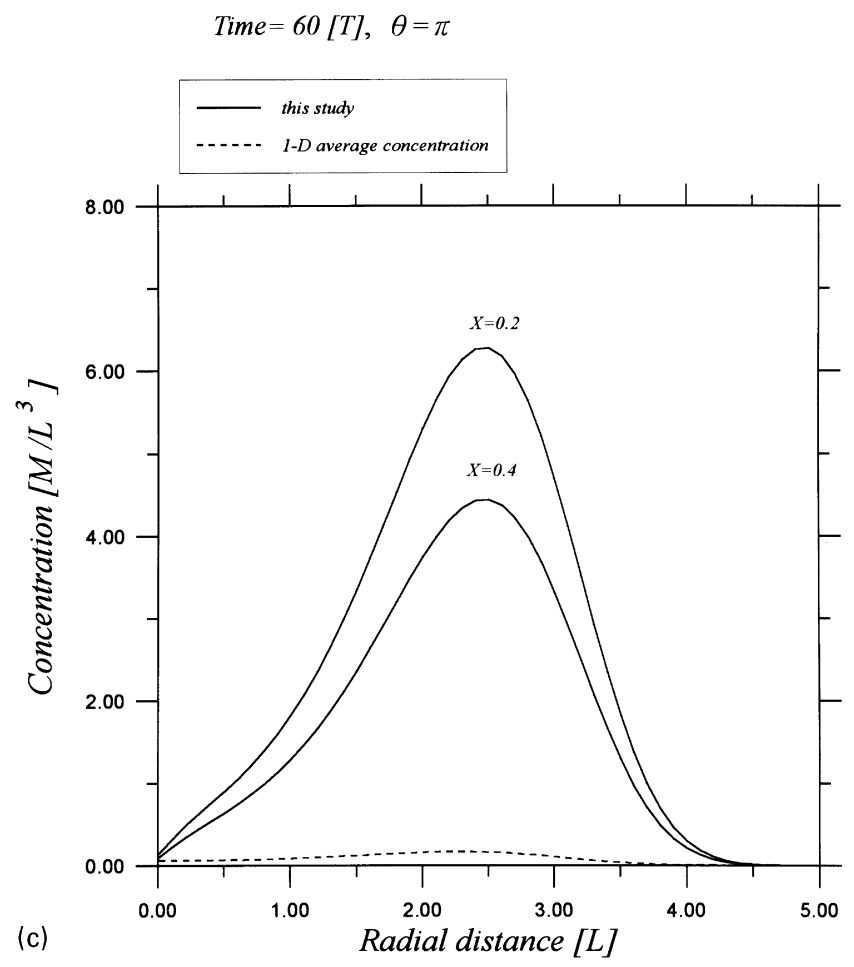

Fig. 9. (Continued).

Fourier cosine inversion on dimensional radial distance and dimensionless dispersivity ratio. Figs. 4(a)-(c) present the dependence for a time equal to $20[\mathrm{~T}]$ and for various Peclet numbers.

\subsection{Case 2}

The second solution verification case is the twodimensional, finite element solution, as employed by Guvanasen and Guvanasen (1987) to verify their approximate solution to the problem of convergent radial dispersion of a tracer in a configuration similar to that in Fig. 2. Fig. 5 illustrates the shape of the initial pulse and the domain discretized by finite elements. The non-axisymmetric transport of a tracer pulse is initially located between $0.98 R$ and $1.02 R$ of radial distance, and transverse angles $\theta=0^{\circ}$ and $\theta=$ $2.5^{\circ}$. The upstream zone is extended to $1.4 R$ from the center of pumping well. Only a sector of $17.5^{\circ}$ is used to represent the entire transport domain in Guvanasen and Guvansen's study (1987) because the finite element solution is valid as long as the tracer concentration has not reached the lateral boundary and the total numbers of nodes can be reduced. Fig. 6 presents the breakthrough curves at four observation points. Fig. 5 illustrates the locations of these points. At all observation points, the finite element and revised LTFD solutions agree well with each other.

Comparing the above analytical and finite element solutions reveals that the revised LTFD model can

Fig. 9. (a) Comparison of longitudinal concentration profiles from the proposed model for dimensionless dispersivity ratios $X=0.2$ and 0.4 , and from the one-dimensional averaging solution $(P e=1)$. (b) Comparison of longitudinal concentration profiles from the proposed model for dimensionless dispersivity ratios $X=0.2$ and 0.4 , and from the one-dimensional averaging solution $(P e=10)$. (c) Comparison of longitudinal concentration profiles from the proposed model for dimensionless dispersivity ratios $X=0.2$ and 0.4 , and from the one-dimensional averaging solution $(P e=100)$. 

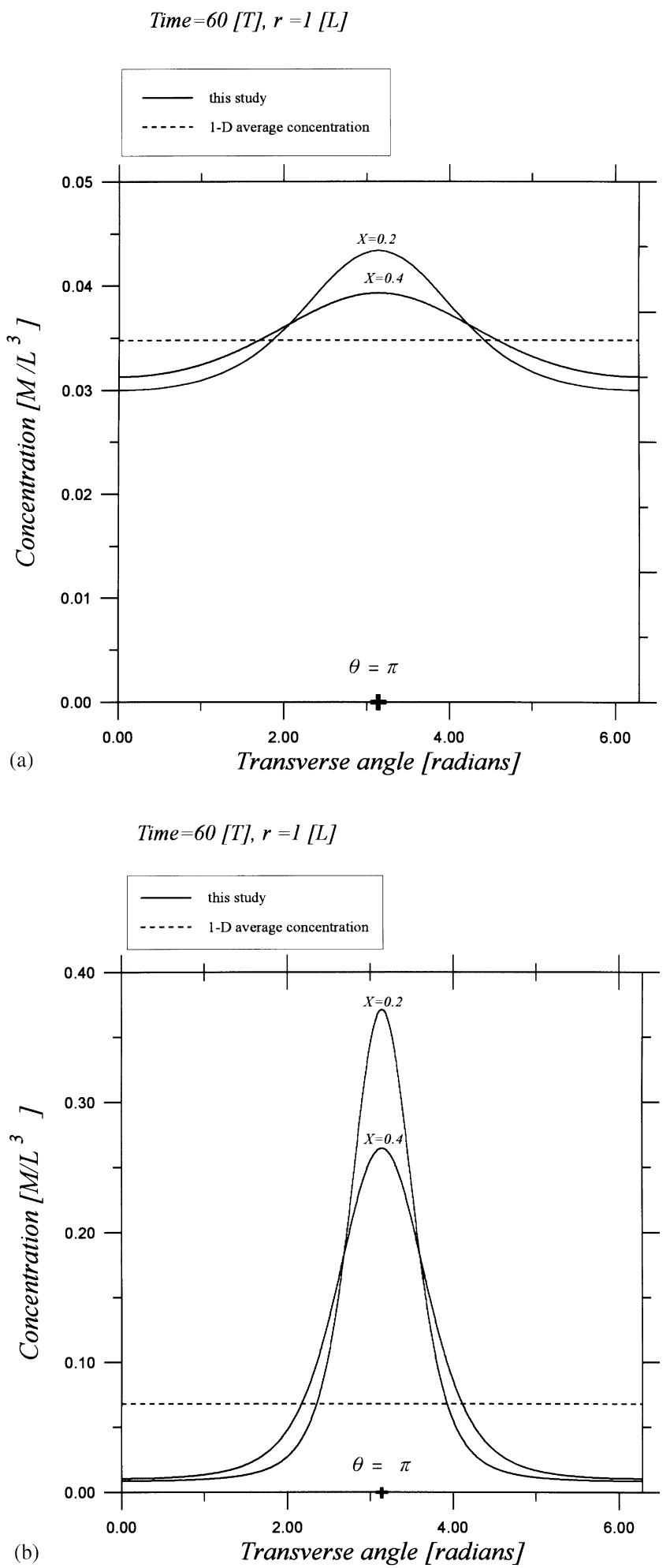


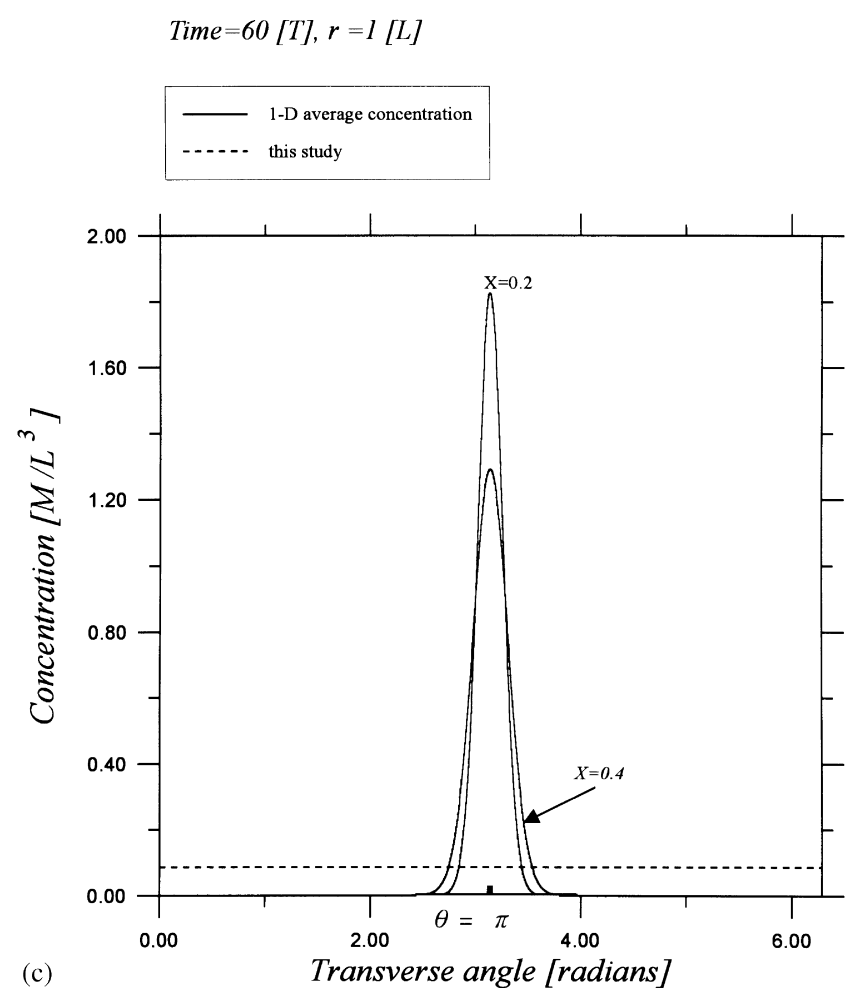

Fig. 10. (Continued).

effectively and accurately handle the radial advection-dispersion equation.

\section{Effect of transverse dispersion}

The verified revised LTFD model is applied to illustrate how transverse dispersion influences tracer transport in a convergent radial tracer experiment. A hypothetical field case as shown in Fig. 7(a) is designed herein to simulate the transport behavior of solute from the injection well into the pumping well, and to analyze the effect of transverse dispersion in a convergent flow field. The input parameters are the same as those for the model verification in Case 1 in the previous section. The steady-state convergent flow field is depicted in Fig. 7(a). The arrows and their adjacent values depict the direction and magnitude of the velocity of groundwater flow. Figs. 7(b)-(f) plot the simulated concentration contours for times 20, 40, 60, 80 and 100 [T] with Peclet number $=$ 100. Initially, the tracer plume spreads in the longitudinal and transverse directions as a circular shape (Fig. 7(b)). Later, owing to the convergent flow field, the tracer plume is pulled by the advection and longitudinal dispersion along the straight line which is connected by the pumping and injection wells. The tracer plume gradually changes to an oval shape (Figs. 7(c) and (d)). Eventually, most of the tracer is drawn into the pumping well and the tracer plume is shrunk to a smaller size, then disappears. The twodimensional concentration contours do not clearly

Fig. 10. (a) Comparison of transverse concentration profiles from the proposed model for dimensionless transverse dispersivity ratios $X=0.2$ and 0.4 , and from one dimensional averaging solution $(P e=1)$. (b) Comparison of transverse concentration profiles from the proposed model for dimensionless transverse dispersivity ratios $X=0.2$ and 0.4 , and from one dimensional averaging solution $(P e=10)$. (c) Comparison of transverse concentration profiles from the proposed model for dimensionless transverse dispersivity ratios $X=0.2$ and 0.4 , and from one dimensional averaging solution $(P e=100)$. 
display how transverse dispersion influences the tracer transport in the radially convergent flow field (Figs. 7(b)-(f)).

To illustrate clearly how transverse dispersion influences the breakthrough curve at any given observation well, both the two-dimensional solution and the one-dimensional averaging solution are constructed. Figs. 8(a)-(c) display the breakthrough curves of the two-dimensional revised LTFD model at $r=1$ [L], $\theta=\pi$ for Peclet numbers of 1,10 and 100 , respectively. The dashed lines denote the breakthrough curves of the average concentration at $r=1$, where no concentration variation occurs in the transverse direction. The average concentrations, $\bar{C}$, are calculated by averaging the tracer concentration from transverse angle $\theta=0$ to $\theta=2 \pi$ at fixed radial distance, $\bar{C}(r, t)=1 / 2 \pi \int_{0}^{2 \pi} C(r, \theta, t) \mathrm{d} \theta$, and then set to be onedimensional solutions. Notably, arrival times of peak concentrations are nearly identical for both the twodimensional solution and the one-dimensional averaging solution. Since the dispersive process only causes spreading of the solute, when transverse dispersion is considered, the solute moves transversely owing to the concentration gradient in the transverse direction. The direction of transverse solute transport is normal to the advection flow path. The total mass of the tracer in a given concentric circle (e.g. the concentric circle of $r=1$ in Fig. 7(a)) area remains unchanged. In addition, the mass conservation in the concentric circle area preserves the arrival time of peak concentration. The transverse dispersion only affects the magnitude of the peak concentration. Figs. 8(a)-(c) also reveal that the peak concentration in two-dimensional solutions exceeds that in a onedimensional averaging concentration. This concentration difference increases with a decrease of dimensionless dispersivity ratio $(X)$ and an increase of Peclet numbers.

To further illustrate how transverse dispersion influences the spatial distribution of the solute, Figs. 9(a)-(c) and 10(a)-(c) display the radial (longitudinal) concentration profiles at $\theta=\pi$ and transverse concentration profiles (concentric circle) at $r=1$ [L] for a time of 60 [T]. Fig. 9(a)-(c) reveals that the two-dimensional concentration curves always exceed the one-dimensional averaging concentration curves. Fig. 10(a)-(c) shows that the transverse concentration profiles are symmetrical around the line connecting the pumping well to the injection well, or at $\theta=\pi$. The maximum concentration in the transverse concentration profile is where $\theta=\pi$. This finding suggests that the transverse dispersion causes the tracer to spread in the transverse direction (or normal to flow direction) and are symmetrically redistributed around $\theta=\pi$. Increasing the transverse dispersivity (dimensionless dispersivity ratio) enhances the spreading of the tracer extensively and decreases the difference between the maximum tracer concentration $(\theta=\pi)$ in a two-dimensional solution and the onedimensional average tracer concentration. Fig. 10(a)(c) also can be used to explain the results shown in Figs. 8(a)-(c) and 9(a)-(c). If the observation well is chosen to be close to the line connecting the pumping well and the injection well, the concentration in two-dimensional solutions exceeds the one-dimensional averaging concentration. Far from the axis, therefore, the twodimensional solution shows a lower concentration than a one-dimensional average solution.

From the above discussion on breakthrough curves and the spatial profile of tracer concentration, we can infer that transverse dispersion spreads the concentration in lateral directions and lowers the peak concentration. However, the arrival time of the peak concentration remains the same as in the one-dimensional solution. This finding also suggests that the different transverse dispersion profiles that depend on the dimensionless dispersivity ratio can be employed to determine the transverse dispersivity of a field tracer test.

\section{Determination of transverse dispersivity}

If the formation thickness, injection tracer mass and pumping rate are known, it is theoretically possible to determine, in a convergent tracer test, the following transport properties: effective porosity, longitudinal dispersivity and lateral dispersivity using the developed two-dimensional solution. By matching the theoretical and observed breakthrough curve at the pumping well, the effective porosity and longitudinal dispersivity can be determined. If an observation is made between the injection well and the pumping well, breakthrough curves registered in this latter well can be utilized to determine the dimensionless dispersivity ratio, the ratio of lateral to longitudinal dispersivity (Guvanasen and Guvanasen, 1987). The 


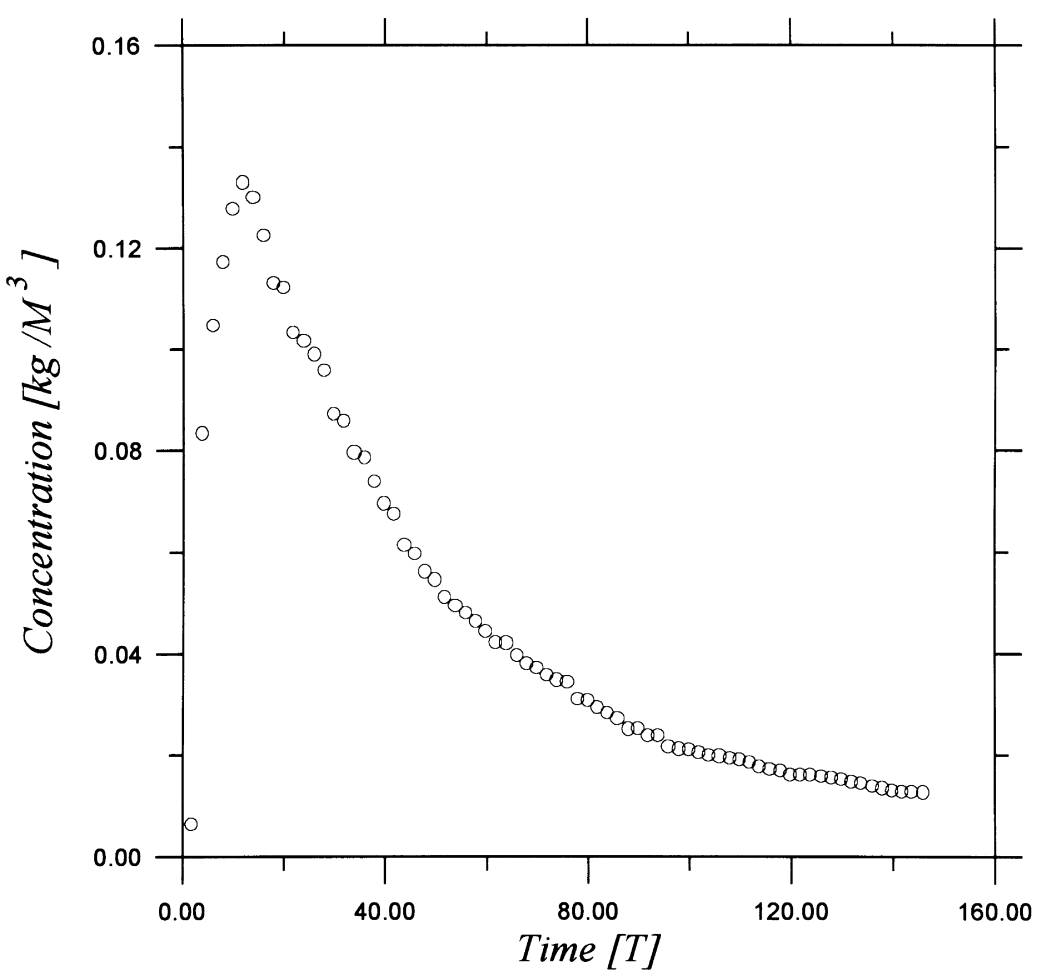

Fig. 11. Hypothetical tracer test data for breakthrough curve at observation at well of $r=1, \quad \theta=\pi$.

curves in Fig. 8(a)-(c) facilitate the determination of transverse dispersivity.

Analysis of the proposed method consists of the following steps:

1. The longitudinal dispersivity and effective porosity are estimated.

(a) The concentrations measured at the pumping well are normalized relative to the peak concentration and are plotted semi-logarithmically against the real time.

(b) The experimental data are matched against the type curves of Moench (1989), and the Peclet numbers are obtained (Sauty, 1980).

(c) The longitudinal dispersivity and effective porosity are calculated from

$a_{\mathrm{L}}=\frac{R}{P e}$

$n_{\mathrm{e}}=\frac{Q t}{\pi R^{2} b t_{\mathrm{D}}}$
2. The transverse dispersivity is estimated.

(a) The experimental concentration observed at other observation well is plotted against real time. (b) The theoretical breakthrough curves for different dimensionless dispersivity ratios at this well are generated from the revised LTFD model for Peclet numbers obtained from step 1.

(c) The experimental breakthrough curves are matched against the theoretical breakthrough curves and the dimensionless dispersivity ratio is obtained. (d) The transverse dispersivity is calculated from $a_{\mathrm{T}}=a_{\mathrm{L}} X$

Next, a hypothetical example is presented to illustrate the analysis according to the proposed method.

\subsection{Example}

Consider a hypothetical tracer test. The distances 


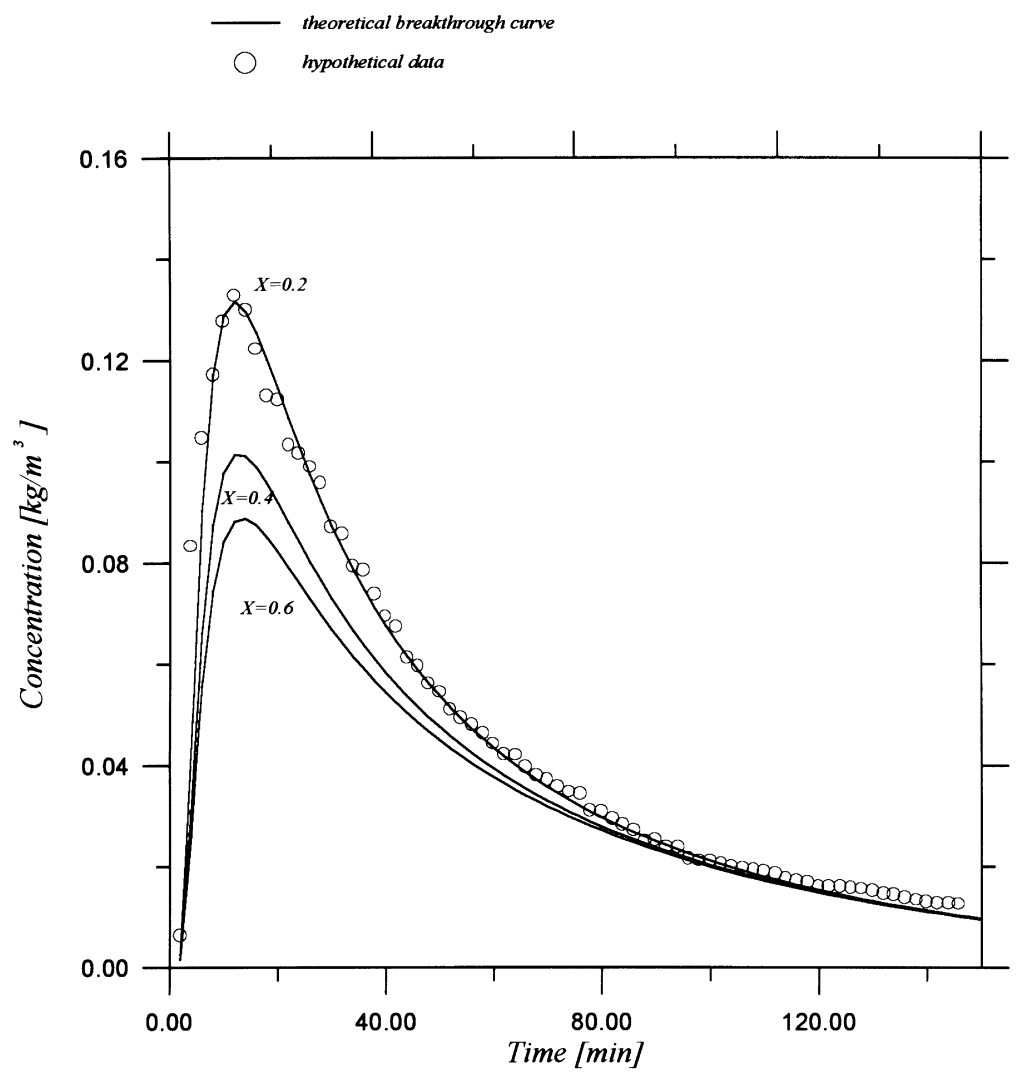

Fig. 12. The hypothetical tracer test data at observation point, $r=1[\mathrm{~L}], \quad \theta=\pi$, matched with the theoretical breakthrough of the revised LTFD model.

between the pumping well and the injection well, $R$, are $5 \mathrm{~m}$. The concentrations measured at the pumping well are normalized relative to the peak concentration. The estimated Peclet number is 1.0 for the matching of the hypothetical data against the type curves. Longitudinal dispersivity is then computed from

$a_{\mathrm{L}}=\frac{R}{P e}=\frac{5.0[\mathrm{~m}]}{1.0}=5.0[\mathrm{~m}]$

Fig. 11 plots the experimental breakthrough curve at the observation at $r=1, \theta=\pi$. The theoretical breakthrough curves at $r=1, \theta=\pi$ as shown in Fig. 8(a)-(c), can be generated from the revised LTFD model for a Peclet number of 1 . As curves are satisfactorily matched, the dimensionless dispersivity ratio can be obtained as shown in Fig.
12. Transverse dispersivity is then computed

$a_{\mathrm{T}}=X a_{\mathrm{L}}=0.2 \times 5[\mathrm{~m}]=1.0[\mathrm{~m}]$

Theoretically, one pumping well and an observation well are enough to determine longitudinal and transverse dispersivity. If concentrations data are available at several intermediate wells, they can be used to cross-check the value of transverse dispersivity or can be interpreted together to obtain a coherent identification and to explain apparent anomalies.

\section{Conclusions}

This work presents a two-well method to evaluate transverse dispersion coefficients for tracer tests in a radially convergent flow field. A revised LTFD model developed on the basis of a conceptual model, 
provides further insight into the transverse spreading of contaminants for a convergent tracer test. Results indicate that the transverse dispersion spreads the concentration in the transverse direction and preserves the arrival time of peak concentration. This finding suggests that different breakthrough curves can be employed to determine the transverse dispersivity of a field tracer test, according to different transverse dispersivities. Then, a curve-fitting method involving a theoretical breakthrough curve, is proposed to evaluate transverse dispersivity. A hypothetical experiment demonstrates the applicability of the proposed model. The two-well method is useful in determining the transverse dispersion coefficient for tracer tests in a radially convergent flow field.

\section{Acknowledgements}

The authors would like to thank the National Science Council of the Republic of China for financially supporting this work under Contract No. NSC 85-2321-B-002-004. Professor Sauty and the other anonymous reviewer are also appreciated for helpful suggestions relating to the determination of transverse dispersivity.

\section{Appendix A}

In this appendix the revised LTFD solution of Eq. (8) subjected to boundary conditions Eqs. (9)-(13) is derived.

Initially proceeding with the Laplace transform of Eq. (8) and its associated boundary conditions (9)(13), with respect to $\tau$, we obtain

$$
\begin{gathered}
\frac{1}{\rho} \frac{\mathrm{d}^{2} G}{\mathrm{~d} \rho^{2}}+\frac{1}{\rho} \frac{\mathrm{d} G}{\mathrm{~d} \rho}+\frac{X}{\rho^{3}} \frac{\partial^{2} C}{\partial \theta^{2}}+C_{R}\left[H\left(\rho+\rho_{\mathrm{w}}\right)\right. \\
\left.-H\left(\rho-\rho_{\mathrm{w}}\right)\right]\left[H\left(\frac{\Delta \theta}{2}\right)-H(0)+H(2 \pi)\right. \\
\left.-H\left(2 \pi-\frac{\Delta \theta}{2}\right)\right]=p G \\
\frac{\mathrm{d} G(\rho, \theta, p)}{\mathrm{d} \rho}=0 \quad \text { at } \rho_{\mathrm{c}}
\end{gathered}
$$

$$
\begin{aligned}
& G(\rho, \theta, p)+\frac{\mathrm{d} G(\rho, \theta, p)}{\mathrm{d} \rho}=0 \quad \rho=\rho_{\mathrm{L}} \\
& \frac{\partial G(\rho, 0, \tau)}{\partial \theta}=\frac{\partial G(\rho, 2 \pi, \tau)}{\partial \theta}=0 \\
& \frac{\partial G(\rho, 0, \tau)}{\partial \theta}=\frac{\partial G(\rho, 2 \pi, \tau)}{\partial \theta}=0
\end{aligned}
$$

where $p$ denotes the Laplace transform parameter and $G$ represents the Laplace transform of $C$, as defined by

$$
\begin{aligned}
G(\rho, \theta, p) & =\int_{0}^{\infty} C(\rho, \theta, \tau) \mathrm{e}^{-p \tau} \mathrm{d} \tau \\
C(\rho, \theta, \tau) & =L^{-1}[G(\rho, \theta, p)] \\
& =\frac{1}{2 \pi \mathrm{i}} \int_{c-i \infty}^{c+i \infty} G(\rho, \theta, p) \mathrm{e}^{-p \tau} \mathrm{d} p
\end{aligned}
$$

Using the finite Fourier cosine transform with respect to $\theta$ of (A1)-(A5), we obtain

$$
\frac{1}{\rho} \frac{\mathrm{d}^{2} W}{\mathrm{~d} \rho^{2}}+\frac{1}{\rho} \frac{\mathrm{d} W}{\mathrm{~d} \rho}-\left(p+\frac{X n^{2}}{\rho^{3}}\right) W=f_{1}(\rho) \bar{g}(n)(\mathrm{A} 8)
$$

where

$$
\begin{aligned}
\bar{g}(n) & =F_{c}[g(\theta)] \\
& =\left\{\begin{array}{c}
\frac{\Delta \theta}{2} \quad \text { if } n=0 \\
\frac{1}{n}\left[\left[\sin (n \pi)-\sin \left(n \pi-\frac{\Delta \theta}{2}\right)\right] \text { if } n\right. \\
\\
>0, n=1,2,3 \ldots
\end{array}\right.
\end{aligned}
$$

The symbol $F_{\mathrm{c}}[]$ represents the finite Fourier cosine transform.

$$
\begin{aligned}
& \frac{\mathrm{d} W(\rho, n, p)}{\mathrm{d} \rho}=0 \quad \text { at } \rho=\rho_{\mathrm{c}} \\
& W(\rho, n, p)+\frac{\mathrm{d} W(\rho, n, p)}{\mathrm{d} \rho}=0 \quad \text { at } \rho=\rho_{\mathrm{L}}
\end{aligned}
$$

where $n$ denotes the finite Fourier cosine transform parameter and $W$ represents the finite Fourier cosine transform of $G$, as defined by

$W(\rho, n, p)=F_{\mathrm{c}}\{G\}=\int_{0}^{\pi} G(\rho, \theta, p) \cos (n \theta) \mathrm{d} \theta(\mathrm{A} 11)$ 
Such a transform is advantageous in that the inversion is directly given by the following formula (Sneddon, 1972):

$$
\begin{aligned}
G(\rho, \theta, p) & =F_{\mathrm{c}}^{-1}[W(\rho, n, p)] \\
& =\frac{1}{\pi} W(\rho, 0, p)+\frac{2}{\pi} \sum_{n=1}^{\infty} W(\rho, n, p) \cos (n \theta)
\end{aligned}
$$

A finite difference method is employed by discretizing the radial distance of the transformed partial differential equation. The advection terms are approximated using the upwind difference formulae. After the difference formulae are substituted into the transformed partial differential equation, the algebraic equation thus obtained takes the following form:

$$
\begin{gathered}
\frac{1}{\rho_{i}} \frac{W_{i+1}-2 W_{i}+W_{i+1}}{\Delta \rho^{2}}+\frac{1}{\rho_{i}} \frac{W_{i+1}-W_{i}}{\Delta \rho} \\
-\left(p+\frac{x n^{2}}{\rho_{i}^{3}}\right) W_{i}=f_{1}\left(\rho_{i}\right) \bar{g}(n)
\end{gathered}
$$

By grouping and rearranging terms, the final form of the finite difference equation in the Laplace-finite Fourier cosine domain is

$a W_{i+1}+b W_{i}+c W_{i-1}=d$

where $\quad a=\rho_{i}^{2}+\rho_{i}^{2} \Delta \rho, \quad b=-2 \rho_{i}^{2}-\rho_{i}^{2} \Delta \rho-$ $\Delta \rho^{2}\left(p p_{i}^{3}+X n^{2}\right), c=\rho_{i}^{2}$, and $d=\rho_{i}^{3} \Delta \rho^{2} f_{1}\left(\rho_{i}\right) \bar{g}(n)$.

Written in matrix notation, the finite difference system of simultaneous equations (A14) becomes

$$
[\Omega][W]=[D]
$$

where $[\Omega]$ denotes the coefficient matrix, $[W]$ represents the vector of the unknown transformed concentration, and $[D]$ is the known right-hand side vector. The system of algebraic equations, as represented by Eq. (A15), can be solved using direct Gaussian elimination or other types of solvers (e.g., iterative types) to yield a value of Laplace-finite Fourier cosine transformed concentration at the node points. In addition, a FORTRAN subroutine DLSACB can be used (see Visual Numerics Inc., 1994). The solutions in original domain $C(\rho, \theta, \tau)$ are the Laplace and finite Fourier cosine inversions of $W_{i}$. For convenience, the finite Fourier cosine inversion is first performed.

$$
\begin{aligned}
C(\rho, \theta, \tau)= & \frac{1}{\pi} L^{-1}\left[W_{i}(0, p)\right] \\
& +\frac{2}{\pi} \sum_{n=1}^{\infty} L^{-1}\left[W_{i}(n, p) \cos (n \theta)\right]
\end{aligned}
$$

Such an infinite series can be straightforwardly evaluated. However, it is important to consider the point at which a sufficient number of terms have been summed. Thus, the series behavior for a large $n$ must be first analyzed and then discussed. For a large $n$, where $b \gg a>c, W_{i}$ can be approximately expressed as

$W_{i} \approx \frac{d}{b} \approx \frac{\rho_{i}^{3} f_{1}\left(\rho_{i}\right) \bar{g}(n)}{p \rho_{i}^{3}+X n^{2}}$

Assuming that we only sum the first, say, $N$ terms, the remainder, $R_{n}$ of the infinite series, is

$$
\begin{aligned}
\left|R_{\mathrm{n}}\right| & \leq \sum_{n=N+1}^{\infty}\left|L^{-1}\left(W_{i}\right)\right| \leq \sum_{n=N+1}^{\infty} L^{-1}\left[\frac{\rho_{i}^{3} f_{1}\left(\rho_{i}\right) \frac{1}{n}}{p \rho_{i}^{3}+X n^{2}}\right] \\
& =\sum_{n=N+1}^{\infty} L^{-1}\left[\frac{f_{1}\left(\rho_{i}\right)}{n\left(p+X n^{2} / \rho_{i}^{3}\right)}\right] \\
& \leq L^{-1}\left[\frac{f_{1}\left(\rho_{i}\right)}{(N+1)\left[p+X(N+1)^{2} / \rho_{i}^{3}\right.}\right] \leq \varepsilon
\end{aligned}
$$

(A18)where $R_{\mathrm{n}}$ denotes the remainder, and $\epsilon$ represents the tolerance error.

Once the solution is obtained, the Laplace transform of the nodal concentrations must be inverted in the Laplace transform domain. Also, the Laplace inverse of (A16) must be determined numerically. A FORTRAN subroutine DINLAP/INLAP (Visual Numerics Inc., 1994) based on the de Hoog et al. (1982) algorithm, is employed to perform the Laplace inversion.

\section{References}

Carrera, J., Walters, G., 1985. Theoretical developments regarding simulation and analysis of convergent flow tracer test. Sandia National Laboratories.

Chen, J.S., Liu, C.W., Chen, C.S., Yeh, H.D., 1996. A Laplace 
transform solution for tracer tests in a radially convergent flow field with upstream dispersion. J. Hydrol. 183, 263-275.

Domenico, P.A., Robbins, G.A., 1984. A dispersion scale effect in model calibrations and field tracer experiments. J. Hydrol. 70, $123-132$.

Guvanasen, V., Guvanasen, V.M., 1987. An approximate semianalytical solution for tracer injection tests in a confined aquifer with a radially converging flow field and finite volume of tracer and chase fluid. Water Resour. Res. 23 (8), 1607-1619.

de Hoog, F.R., Knight, J.H., Stokes, A.N., 1982. An improved method for numerical inversion of Laplace transforms. SIAM J. Sci. Stat. Comput. 3 (3), 357-366.

Leij, F.J., Dane, J.H., 1991. The effect of transverse dispersion on solute transport in soils. J. Hydrol. 122, 407-422.

Moench, A.F., 1989. Convergent radial dispersion: a Laplace transform solution for aquifer tracer testing. Water Resour. Res. 25 (3), 439-447.

Moltyaner, G.T., Killey, R.W.D., 1988. Twin lake tracer test: transverse dispersion. Water Resour. Res. 24 (10), 16281637.

Moridis, G.J., Reddell, D.L., 1991. The Laplace transform finite difference method for simulation of flow through porous media. Water Resour. Res. 27 (8), 1873-1884.

Sauty, J.-P., 1980. An analysis of hydrodispersive transfer in aquifers. Water Resour. Res. 16 (1), 145-158.
Sneddon, I.N., 1972. The Use of Integral Transform, McGraw-Hill, New York, 539 pp.

Sudicky, E.A., 1989. The Laplace transform Galerkin technique: a time-continuous finite element theory and application to mass transport in groundwater. Water Resour. Res. 25 (8), 18331846.

Sudicky, E.A., Cherry, J.A., 1979. Field observations of tracer dispersion under natural flow conditions in an unconfined sandy aquifer. Water Pollut. Res. Can. 14, 1-17.

Sudicky, E.A., McLaren, R.G., 1992. The Laplace transform Galerkin technique for large-scale simulation of mass transport in discretely fracture porous formations. Water Resour. Res. 28 (2), 499-513.

Sudicky, E.A., Cherry, J.A., Frind, E.O., 1983. Migration of contaminants at a landfill: a case study. 4. A natural-gradient dispersion test. J. Hydrol. 63, 81-108.

Visual Numerics, Inc., 1994. IMSL User's Manual, Houston, TX, vol. 1, pp. 159-161.

Visual Numerics, Inc., 1994. IMSL User's Manual. Houston, TX, vol. 2, pp. 827-830.

Wang, H.Q., Crampon, N., 1995. Method for interpreting tracer experiments in radial flow using modified analytical solutions. J. Hydrol. 165, 11-31. 